\title{
Hedging options in the incomplete market with stochastic volatility*
}

\author{
Rituparna SEN ${ }^{\dagger}$
}

We show that it is possible to avoid the discrepancies of continuous path models for stock prices and still be able to hedge options if one models the stock price process as a birth and death process. One needs the stock and another market traded derivative to hedge an option in this setting. However, unlike in continuous models, the number of extra traded derivatives required for hedging does not increase when the intensity process is stochastic. We obtain parameter estimates using Generalized Method of Moments and describe the Monte Carlo algorithm to obtain option prices. We show that one needs to use filtering equations for inference in the stochastic intensity setting. We present real data applications to study the performance of our modeling and estimation techniques.

AMS 2000 SUBJECT Classifications: 62P05, 62M20, 60G44, 60F05.

KEYWORDS AND PHRASES: Birth and death process, Bayesian filtering, Edgeworth expansion, Convergence of stochastic processes, Generalized method of moments, Monte Carlo algorithm, Hidden Markov model.

\section{INTRODUCTION}

A major difficulty on the interface between statistics and finance is that diffusion models are not really valid descriptions of data when it comes to microstructure. Diffusion models, specially variants of the geometric Brownian motion of Black and Scholes (1973), are the most common models used in finance for stock price processes. The difference between the natural discreteness of the movement of the stock prices determined by the tick size and the assumed continuity in the diffusion model setting can lead to, not only serious mispricing of options, but other conceptual inconsistencies.

The main feature of this inconsistency is that the microstructure predicted by diffusion models includes observable quadratic variation (and hence volatilities), whereas this is nowhere nearly true in practice. According to continuous path models, the integrated volatility equals the

*The research was supported by NSF grants DMS 99-71738 and DMS 02-04639.

${ }^{\dagger}$ The author thanks Per A. Mykland for his valuable advice and guidance. The author is also grateful to Kenneth Wilder for help in obtaining and analyzing the data. quadratic variation. Hence, if data are observed continuously, the volatility should be observable. In practice we only observe a sample of the continuous time path. As shown in, for example, Jacod and Shiryaev (2003), the difference between the quadratic variation at discrete and continuous time scales converges to zero as the sampling interval goes to zero. Hence, the best possible estimates of integrated volatility should be the observed quadratic variation computed from the highest frequency data obtainable. However, it has been found empirically that there is a bigger bias in the estimate when the sampling interval is quite small. For references in this area see Andersen et al. (2001).

On the other hand there is a reason why continuous models are used. These are more or less the only models where one can hedge options. Practitioners are interested not only in pricing options but also in hedging, that is to remove the risks associated with options by trading other instruments. Technically, to hedge means to create self financing replicating portfolios for derivative securities. There has been some interesting work on asymptotic or optimal hedging in the discontinuous price setting as in Hayashi (2005), Hubalek et al. (2006), Mykland (2000) and Schweizer (1992), but not on perfect hedging.

Hence, whereas from a data description point of view, it would make sense to use models with jumps, from a hedging standpoint, these models cannot be used. One of the consequences of this conflict is that statistical information is not used as much as it should be when it comes to valuing derivative securities. Instead there is a substantial reliance on implied quantities, as in Engle and Mustafa (1992), Cont (2002). It is shown in Mykland (1996) that this disregard for historical data can lead to mispricing. It would be desirable to bring as much statistical information as possible to bear on financial modeling and at the same time be able to hedge.

This is where birth and death processes become useful. Birth and death processes have the virtue that one can quite successfully set up derivative securities hedging in this model. It is not quite as straight forward as the continuous model. However, this is still a much nicer situation than what is the case in general models with jumps where it is impossible to hedge. In a sense birth and death processes are almost continuous, as one needs to traverse all intermediate states to go from one point to the other. On the other hand, these processes have a microstructure which conflicts much 
less with the data. In particular, the predictable quadratic variation is not observable.

Another conceptual inconsistency of the continuous models is that the Black-Scholes formula is based on a derivation where all risk is removable from an option position by a continuously rebalanced delta hedge. However, in practice, delta hedging needs to be augmented with gamma hedging and sometimes even vega hedging, which is adhoc and inconsistent with the underlying theory. The need for delta hedging, however, is consistent with the birth and death model as demonstrated in Dengler and Jarrow (1997).

In the current paper we take up the idea of using birth-and-death processes as stock price models as done in Korn et al. (1998), but generalize the existing literature in various ways: First, we use a jump intensity which is proportional to the square of the stock price, a fact that leads to a geometric Brownian motion limit as the jump size goes to zero. Second, the case of a stochastic jump intensity is also considered. Third, the models are applied to real data to demonstrate their pros and cons in application. The main contribution of the paper is to show that the proposed incomplete model allows for both pricing and hedging of options. More on modeling and estimation in this class of models can be found in Russell and Engle (2005) and Large (2005).

The paper is organized as follows. In Section 2 we present the basic model with linear and quadratic jump intensity and the set-up for stochastic intensity rate. Section 3 describes the pricing of options and presents an Edgeworth expansion for option prices. Section 3 describes hedging of options in the constant intensity case. We then go on to derive the hedging strategy in the stochastic intensity case by first conditioning on the unobserved intensity process and then taking the expectation. In Section 5 we study the problem of estimation of model parameters. In the stochastic intensity case, we present a Bayesian solution to the problem and possible generalizations. The prior on the intensity rate process that we study in detail is a two state Poisson jump process. In Section 6 we study the performance of the model in some real data applications and in Section 7 we present the conclusions.

\section{THE MODEL}

\subsection{The linear jump intensity model}

The problem of pricing and hedging options in birth and death models where the intensity of jumps is proportional to the present stock price (linear jump intensity) is solved in Korn et al. (1998). We shall call the constant of proportionality rate. This is the discrete state-space version of the popular affine jump diffusion models, for example see Duffie et al. (2000). More precisely, suppose the stock price $S_{t}$ is a pure jump process with jumps of size $\pm c$. This implies that the process moves on a grid of resolution $c$ and $N_{t}=S_{t} / c$ is a birth and death process. The jumps of the
$N_{t}$ process have random size $Y_{t}$ which is a binary variable taking values \pm 1 and the probability that $Y_{t}=1$ is denoted by $p_{t, N_{t}}$. Suppose that there is a risk-free interest rate $r_{t}$ and the intensity of jumps is $N_{t} \lambda_{t}$ where the rate $\lambda_{t}$ is a non-negative stochastic process. In order to keep the price process away from zero, introduce the condition:

$$
\begin{gathered}
\text { When } N_{t}=1, Y_{t} \text { takes values } 0 \text { and } 1 \\
\text { with probabilities } p_{t, 1} \text { and } 1-p_{t, 1}
\end{gathered}
$$

It is shown in Sen (2006) that when the tick size goes to zero, the price process converges almost surely to a deterministic processs. Hence this is not a very interesting model. However if the intensity of jumps is allowed to be proportional to the square of the stock price, then such a process can be considered as a discretized version of the geometric Brownian motion model for stock prices that corresponds to the popular Black-Scholes theory of asset pricing. This will be called the quadratic jump intensity model. In the following Section this model and the asymptotic result stated above will be formalized. A similar model has been studied in Dengler and Jarrow (1997) where the authors consider a birth and death process on the log of stock prices. This makes the problem technically much easier, but contradicts the fact that stock prices, and not log stock prices, move in multiples of the tick size.

\subsection{The quadratic jump intensity model}

In the same setting as above, we now take the intensity of jumps to be $N_{t}^{2} \lambda_{t}$ where $\lambda_{t}$ is constant or a time-varying deterministic process. Quadratic models have been used successfully in asset pricing as in Lieppold (2002). Piazzesi (2001) includes jumps with quadratic arrival intensity for pricing bonds in the quadratic class.

We have the following result, the proof of which is given in Appendix A.

Theorem 2.1. Let $N_{t}^{(n)}$ be an integer valued jump process. The jump time $\xi_{t}^{(n)}$ follow a counting process with rate $N_{t}^{(n) 2} \sigma_{t}^{2}$. The random jump size $Y_{t}^{(n)}$ is a binary variable taking values \pm 1 and probability that $Y_{t}=1$ is $p_{t, N_{t}}$. Assume that condition (2.1) and assumptions (2.2) and (2.3) are satisfied. Then $X_{t}^{(n)}=\ln \left(N_{t}^{(n)} / n\right)$ converges in distribution to $X_{t}$, a continuous Gaussian Martingale with characteristics $\left(\int_{0}^{t}\left(r_{u}-\sigma_{u}^{2} / 2\right) d u, \int_{0}^{t} \sigma_{u}^{2} d u, 0\right)$ if $p_{t, N_{t}}=\left(r_{t} / N_{t} \sigma_{t}^{2}+1\right) / 2$ and $p_{t, 1}=\left(r_{t}-\sigma_{t}^{2} / 2\right) /\left(\sigma_{t}^{2} \log (2)\right)$.

The assumptions are:

$$
\begin{aligned}
& E \sum_{\tau_{i} \leq t}\left(\frac{1}{N_{\tau_{i}}^{(n)}}\right)^{k} \text { goes to zero as } \\
& n \text { goes to infinity for all } k \geq 4
\end{aligned}
$$

$$
\int_{0}^{t} N_{u}^{2} \sigma_{u}^{2} d u \text { is finite a.s. }
$$



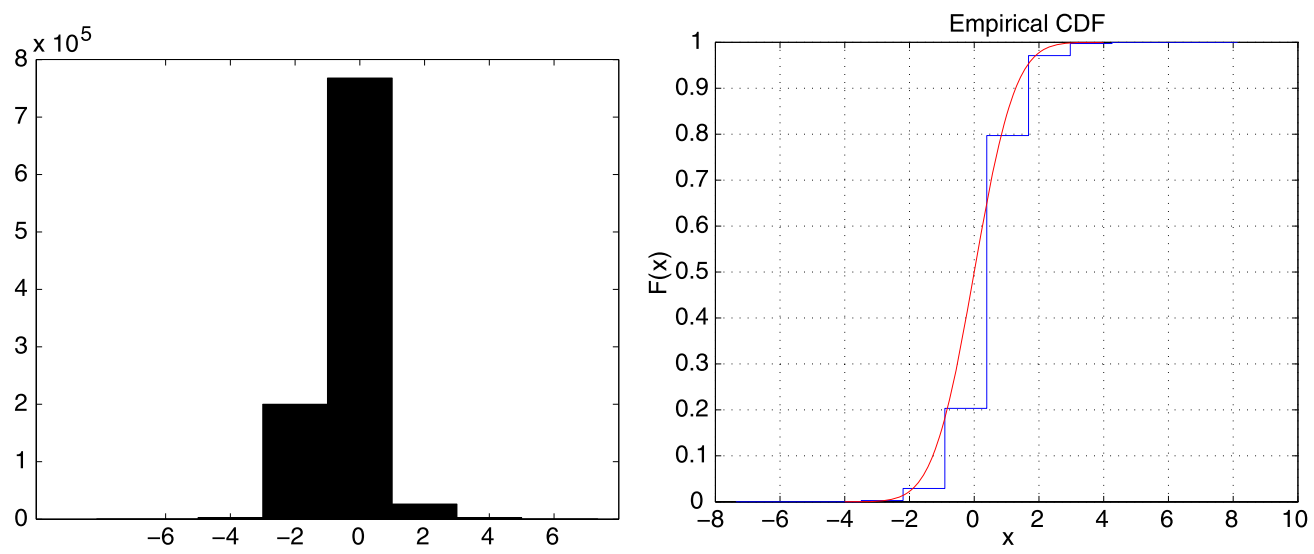

Figure 1. (a) Histogram. (b) Empirical cumulative distribution function superimposed with normal distribution.

Suppose for each $n$, the stock price $S_{t}^{(n)}=N_{t}^{(n)} / n$ where the process $N_{t}^{(n)}$ is described in Theorem 2.1. So the grid size $c$ is $1 / n$. Assume initial stock price $S_{0}^{(n)}$ is the same for all $n$. As $n \rightarrow \infty$, by Theorem 2.1, the sequence of random processes $X_{t}^{(n)}=\ln \left(S_{t}^{(n)}\right)$ converge in distribution to $X_{t}$, a continuous Gaussian Martingale with characteristics $\left(\int_{0}^{t}\left(r_{u}-\sigma_{u}^{2} / 2\right) d u, \int_{0}^{t} \sigma_{u}^{2} d u, 0\right)$. Since exp is a continuous function, $S_{t}^{(n)}=\exp \left(X_{t}^{(n)}\right)$ converge in law to $S_{t}=\exp \left(X_{t}\right)$. The stochastic differential equation of $X$ is:

$$
d\left(X_{t}\right)=\left(r_{t}-\frac{1}{2} \sigma_{t}^{2}\right) d t+\sqrt{\sigma_{t}^{2}} d W_{t}
$$

where $W_{t}$ is standard Wiener process. By Ito's formula,

$$
\begin{aligned}
d\left(S_{t}\right) & =d\left(\exp \left(X_{t}\right)\right) \\
& =S_{t}\left[\left(r_{t}-\frac{1}{2} \sigma_{t}^{2}\right) d t+\sqrt{\sigma_{t}^{2}} d W_{t}\right]+\frac{1}{2} S_{t} \sigma_{t}^{2} d t \\
& =S_{t} r_{t} d t+S_{t} \sqrt{\sigma_{t}^{2}} d W_{t}
\end{aligned}
$$

Thus the limiting distribution is geometric Brownian motion.

Although the process converges to geometric Brownian motion in the limit, the exact process, depending on the values of the parameters, can be far from the limiting process. For e.g., if the stock price is $\$ 100$, tick size is 1 cent, $\lambda=10^{-6}$ and interest rate is $5 \%$, then the distribution of log returns after an hour, obtained by simulating data from the process for $10^{6}$ observations has skewness -0.04 and kurtosis of 4.63. We present the histogram and empirical cumulative distribution function of this simulated dataset in Fig. 1. The Jarque-Bera test statistics for goodness-of-fit to a normal distribution is 112910 while the critical value at $1 \%$ level is 9.21 . Thus the log returns are not normal, as predicted from Black-Scholes model, for finite tick-size and finite time horizon. The skewness is not very large, but there is a large excess kurtosis which supports the heavytailedness observed in real data.
We now show that under this model the market is incomplete. Let $\mathcal{P}_{\lambda}$ denote the probability distribution associated with a birth and death process $N_{t}$ with rate $N_{t}^{2} \lambda$ and probability of birth $p_{t, N_{t}}=\left(r_{t} / N_{t} \lambda+1\right) / 2$. In Theorem 2.2 we show that for any $\tilde{\lambda}$, the measure $\mathcal{P}_{\lambda}$ and $\mathcal{P}_{\tilde{\lambda}}$ are equivalent and the discounted stock price is a $\mathcal{P}_{\tilde{\lambda}}$ martingale. Thus there are infinitely many equivalent martingale measures and hence the market is incomplete.

Theorem 2.2. For any $\tilde{\lambda}$, the probability measures $\mathcal{P}_{\tilde{\lambda}}$ and $\mathcal{P}_{\lambda}$ are mutually absolutely continuous and the discounted security price is a $\mathcal{P}_{\tilde{\lambda}}$ martingale.

Proof. Note that all birth-death processes are supported on the class of step functions that are right continuous and have left limits (r.c.l.l.) with jumps of size \pm 1 on the non-negative integers. Uniqueness of the associated measures corresponding to a jump intensity rate and probability of birth is a consequence of e.g. Theorem 18.4/5 in Lipster and Shiryaev (1978). Hence the measures associated with all birth death processes are mutually absolutely continuous. As a consequence of Theorem 19.7 of Lipster and Shiryaev (1978) we can even give the explicit form of $\mathcal{P}_{\tilde{\lambda}}$ via its Radon-Nikodym derivative with respect to $\mathcal{P}$ as:

$$
\begin{aligned}
\log \frac{d \mathcal{P}_{\tilde{\lambda}}}{d \mathcal{P}}= & \int_{0}^{T} \ln \left(\frac{\tilde{\lambda}_{t} \tilde{p}_{t, N_{t}}}{\lambda_{t} p_{t, N_{t}}}\right) d N_{1 t} \\
& +\int_{0}^{T} \ln \left(\frac{\tilde{\lambda}_{t}\left(1-\tilde{p}_{t, N_{t}}\right)}{\lambda_{t}\left(1-p_{t, N_{t}}\right)}\right) d N_{2 t} \\
& -\int_{0}^{T}\left(\tilde{\lambda}_{t}-\lambda_{t}\right) N_{t}^{2} d t
\end{aligned}
$$

where $d N_{1 t}=I_{Y_{t}=+1} d N_{t}$ and $d N_{2 t}=-I_{Y_{t}=-1} d N_{t}$ with $N_{i 0}=0 . N_{1 t}, N_{2 t}$ are point processes with intensity $\lambda_{t} p_{t, N_{t}} N_{t}^{2}$ and $\lambda_{t}\left(1-p_{t, N_{t}}\right) N_{t}^{2}$ respectively and $d N_{t}=$ $d N_{1 t}-d N_{2 t}$. Let $\tilde{\lambda}_{1 t}=\tilde{\lambda} \tilde{p}_{t, N_{t}} N_{t}^{2}$ and $\tilde{\lambda}_{2 t}=\tilde{\lambda}\left(1-\tilde{p}_{t, N_{t}}\right) N_{t}^{2}$. Then $Q_{i t}=N_{i t}-\int_{0}^{t} \tilde{\lambda}_{i s} N_{s}^{2} d s$ is the compensated point pro- 
cess associated with $N_{i}$ under $\mathcal{P}_{\tilde{\lambda}}$.

$$
\begin{aligned}
d N_{t} & =d N_{1 t}-d N_{2 t} \\
& =d Q_{1 t}-d Q_{2 t}+\left(\tilde{\lambda}_{1 t}-\tilde{\lambda}_{2 t}\right) N_{t}^{2} d t \\
& =d Q_{1 t}-d Q_{2 t}+\left(2 \tilde{p}_{t, N_{t}}-1\right) \tilde{\lambda}_{t} N_{t}^{2} d t \\
& =d Q_{1 t}-d Q_{2 t}+r_{t} N_{t} d t
\end{aligned}
$$

So $\exp \left\{-\int_{0}^{t} r_{s} d s\right\} N(t)$ is a martingale with respect to $\mathcal{P}_{\tilde{\lambda}}$.

\subsection{Stochastic intensity rate}

The intensity rate of jump processes is analogous to volatility in continuous models. Both theoretical and empirical considerations support the need for stochastic volatility. Asset returns have been modeled as continuous processes with stochastic volatility as in Hull and White (1987), Naik (1993) or as jump processes with stochastic volatility as in Bates (1996), Duffie et al. (2000).

So, next we consider the case that the unobserved intensity rate $\lambda_{t}$ being a stochastic process and $N_{t}$ is a birth and death process conditional on the $\lambda_{t}$ process. This can be formally carried out by letting $N_{t}$ be the integral of the $Y_{t}$ process with respect to the random measure that has intensity $\lambda_{t} N_{t}^{2}$ (see, e.g., Ch. II.1.d(p. 71-74) of Jacod and Shiryaev (2003)).

We first put a two state Markov model on $\lambda_{t}$. This assumes that stock price movements fluctuate between low and high intensity rate regimes. This is the approach in Naik (1993). In Section 4.3 we study the hedging strategy for options in this setting. In Section 5.3 we generalize this to any given model on the intensity rate process. For example, alternatively we can consider cases where the intensity rate follows a diffusion as in Hull and White (1987).

\section{PRICING OF OPTIONS}

For any $\lambda$, let $\mathcal{P}_{\lambda}$ be the measure associated with a birthdeath process with event rate $\lambda N_{t}^{2}$ and probability of birth $p_{t, N_{t}}=\left(1+r_{t} / \lambda N_{t}\right) / 2$. Let us denote the class of these measures by $\mathbb{P}$. We have seen in Theorem 2.1 that as the tick size goes to zero, this process converges to geometric Brownian motion. We now restrict our attention to the case of fixed tick-size.

We have seen in Theorem 2.2 that the $\mathcal{P}_{\lambda}$ 's are equivalent and the discounted stock price is a martingale under each such measure. From the fundamental theorem of asset pricing, there exists a measure, called the risk-neutral measure, that is equivalent to the physical measure and under which prices of all traded assets are martingales. Let us assume that the risk neutral measure belongs to $\mathbb{P}$. From this point on we shall work with the risk-neutral measure. The price, at time $t$, associated with any integrable contingent claim with terminal payoff $P$ at time $T$ is $\mathrm{E}\left(\exp \left\{-\int_{\mathrm{t}}^{\mathrm{T}} \mathrm{r}_{\mathrm{s}} \mathrm{ds}\right\} \mathrm{P} \mid \mathcal{F}_{\mathrm{t}}\right)$.

\subsection{Edgeworth expansion for option prices}

Option prices are expectations, under the model, of discounted terminal payoffs. It is of interest to see how much these expectations differ from expectation under the Brownian motion model. Let us define

$$
\begin{aligned}
& X_{t}^{(n)}= \ln \left(\frac{N_{t}^{(n)}}{n}\right) \\
& X_{t}^{*(n)}= X_{t}^{(n)}-X_{0}^{(n)} \\
&-\int_{0}^{t}\left[p_{u, N_{u}} \ln \left(1+\frac{1}{N_{u}}\right)\right] N_{u}^{2} \sigma_{u}^{2} d u \\
&+\int_{0}^{t}\left[\left(1-p_{u, N_{u}}\right) \ln \left(1-\frac{1}{N_{u}}\right)\right] N_{u}^{2} \sigma_{u}^{2} d u \\
& \text { where } \quad p_{t, N_{t}}=\frac{1}{2}\left(1+\frac{r_{t}}{N_{t} \sigma_{t}^{2}}\right)
\end{aligned}
$$

Let $\mathcal{C}$ be the class of functions $g$ that satisfy the following: (i) $\int|\hat{g}(x)| d x<\infty$, uniformly in $C$, and $\left\{\sum_{u} x_{u}^{2} \hat{g}(x), g \in C\right\}$ is uniformly integrable (here, $\hat{g}$ is the Fourier transform of $g$, which must exist for each $g \in C)$; or (ii) $g(x)=f\left(z^{i} x_{i}\right)$, with $\sum_{i} z^{i} z^{i}, f$ and $f^{\prime \prime}$ bounded, uniformly in $\mathcal{C}$, and with $\left\{f^{\prime \prime}: g \in \mathcal{C}\right\}$ equicontinuous almost everywhere (under Lebesgue measure). Under assumptions (I1) and (I2) stated in Appendix B, for any $g \in \mathcal{C}$,

$$
\mathrm{E} g\left(X_{T}^{*(n)}\right)=\mathrm{E} g(N(0, \lambda T))+o(1 / n)
$$

The details are given in Appendix B. Thus, if the payoff function has some smoothness properties, then the expectation under the birth and death model is very close to that under the geometric Brownian motion model. However, many traded options do not have these smoothness properties. That is why we study these deviations computationally in Section 6.

\section{HEDGING}

\subsection{Background}

To hedge an option means to form a self-financing replicating portfolio for the option. Self-financing means we do not have to put in money into the portfolio after the initial time. Replicating means that the terminal value of this portfolio is the same as the payoff of the option. That is, we would like to arrange things so that there is absolutely no risk to us of having to pump in any of our own money later to cover the option at expiration.

We form a self-financing risk-less portfolio with the stock, the bond and two options. Risk-less means that there is no randomness involved in the total value of the portfolio. For this portfolio, we obtain the hedge ratios, that is, the proportion of money invested in each asset at each point of time. The idea is similar to that of delta-neutral hedges 
in stochastic volatility models as discussed in Bakshi et al. (1997).

In this section we derive the hedging strategy in this market. We can hedge an option by trading the stock, the bond and another option.

\subsection{Hedging when the intensity rate is constant}

Let $F_{1}(x, t), F_{2}(x, t)$ be the prices of two options $\mathcal{O}_{1}$ and $\mathcal{O}_{2}$ at time $t$ when the price of the stock is $c x$. Let $F_{3}(x, t)=$ $c x$ be the price of the stock and $F_{0}(x, t)=B_{0} \exp \left\{-\int_{0}^{t} r_{s} d s\right\}$ be the price of the bond. Note that $N_{t}$ is the difference of two counting processes: $N_{0 t}$, the number of births and $N_{1 t}$, the number of deaths. Assume $F_{i}$ are continuous in both arguments and the following partial derivatives exist.

$$
\frac{\partial F_{i}}{\partial t}=\alpha_{F_{i}} \quad \frac{\partial F_{i}}{\partial N_{0}}=\beta_{F_{i}} \quad \frac{\partial F_{i}}{\partial N_{1}}=\gamma_{F_{i}}
$$

We shall construct a self financing risk-less portfolio

$$
V(t)=\sum_{i=0}^{3} \phi^{(i)}(t) F_{i}(x, t)
$$

Let $u^{(i)}(t)=\frac{\phi^{(i)}(t) F_{i}(x, t)}{V(t)}$ be the proportion of wealth invested in asset $i$.

$$
\sum u^{(i)}=1
$$

Since $V_{t}$ is self financing,

$$
\begin{aligned}
\frac{d V(t)}{V(t)}= & \sum_{i=0}^{3} u^{(i)}(t) \frac{d F(x, t)}{F(x, t)} \\
= & u^{(0)}(t) r_{t} d t+u^{(3)}(t) \frac{1}{c x}\left(d N_{0 t}-d N_{1 t}\right) \\
& +\sum_{i=1}^{2} u^{(i)}(t) \alpha_{F_{i}}(x, t) d t \\
& +\sum_{i=1}^{2} u^{(i)}(t)\left(\beta_{F_{i}}(x, t) d N_{0 t}+\gamma_{F_{i}}(x, t) d N_{1 t}\right)
\end{aligned}
$$

$V_{t}$ is risk-less implies

$$
\begin{array}{r}
u^{(3)}(t) \frac{1}{c x}+\sum_{i=1}^{2} u^{(i)}(t) \beta_{F_{i}}(x, t)=0 \\
-u^{(3)}(t) \frac{1}{x}+\sum_{i=1}^{2} u^{(i)}(t) \gamma_{F_{i}}(x, t)=0
\end{array}
$$

The no arbitrage assumption implies

$$
u^{(0)}(t) r_{t}+\sum_{i=1}^{2} u^{(i)}(t) \alpha_{F_{i}}(x, t)=r_{t}
$$

Solving equations (4.1)-(4.4), we get the hedge ratios as:

$$
\begin{aligned}
& u^{(1)}=\left[\left(1-\frac{\alpha_{F_{1}}}{r}-x \beta_{F_{1}}\right)-\frac{\gamma_{F_{1}}+\beta_{F_{1}}}{\gamma_{F_{2}}+\beta_{F_{2}}}\left(1-\frac{\alpha_{F_{1}}}{r}-x \beta_{F_{1}}\right)\right]^{-1} \\
& u^{(2)}=\left[\left(1-\frac{\alpha_{F_{2}}}{r}-x \beta_{F_{2}}\right)-\frac{\gamma_{F_{2}}+\beta_{F_{2}}}{\gamma_{F_{1}}+\beta_{F_{1}}}\left(1-\frac{\alpha_{F_{2}}}{r}-x \beta_{F_{2}}\right)\right]^{-1} \\
& u^{(0)}=-\frac{1}{r_{t}}\left(u^{(1)} \alpha_{F_{1}}+u^{(2)} \alpha_{F_{2}}\right) \\
& u^{(3)}=-x\left(u^{(1)} \beta_{F_{1}}+u^{(2)} \beta_{F_{2}}\right)
\end{aligned}
$$

A self-financing replicating portfolio for option $\mathcal{O}_{2}$ can be formed by investing $\phi^{(i)} / \phi^{(2)}$ units of asset $i$, that is, $u^{(0)} F^{(2)} / u^{(2)} F^{(0)}$ units of Bond, $u^{(3)} F^{(2)} / u^{(2)} F^{(3)}$ units of Stock and $u^{(1)} F^{(2)} / u^{(2)} F^{(1)}$ of option $\mathcal{O}_{1}$.

\subsection{Hedging when the intensity rate is time varying and stochastic}

Let us assume that $\lambda_{t}$ is a Markov process with state space $\left\{\lambda_{0}, \lambda_{1}\right\}$. Suppose there is an unobserved state process $\theta_{t}$ which takes 2 values, say 0 and 1 . The transition matrix is Q. When $\theta_{t}=i, \lambda_{t}=\lambda_{i}$. Jump process associated with $\theta_{t}$ is $\zeta_{t}$. We denote by $\left\{\mathcal{G}_{t}\right\}$ the complete filtration $\sigma\left(S_{u}, \lambda_{u}, 0 \leq\right.$ $u \leq t)$.

We introduce $\pi_{i}(t)=P\left(\theta_{t}=i \mid \mathcal{F}_{t}\right)$ where $\mathcal{F}_{t}=\sigma\left(S_{u}, 0 \leq\right.$ $u \leq t)$.

Let $G_{i}(x, t):=E\left(P \mid \mathcal{G}_{t}\right)$ for a security with payoff $P$ when the stock price is $c x$ and $\lambda_{t}=\lambda_{i}$.

Let $G(x, t):=E\left(P \mid \mathcal{F}_{t}\right)=\pi_{0}(t) G_{0}(x, t)+\pi_{1}(t) G_{1}(x, t)$.

As shown in Snyder (1973), the $\pi_{i t}$ process evolves as:

$$
d \pi_{0 t}=a(t) d t+b(t, 0) d N_{0 t}+b(t, 1) d N_{1 t}
$$

where $a(t)$ and $b(t, i)$ are $\mathcal{F}_{t}$ adapted processes. Note that $\pi_{1 t}=1-\pi_{0 t}$.

Theorem 4.1. $d G / G=\tilde{\alpha_{G}} d t+\tilde{\beta_{G}} d N_{0 t}+\tilde{\gamma_{G}} d N_{1 t}$ where

$$
\begin{aligned}
\tilde{\alpha_{G}=} & \frac{a(t)\left(G_{0}-G_{1}\right)-\left(\pi_{0 t} \frac{\partial G_{0}}{\partial t}+\pi_{1 t} \frac{\partial G_{1}}{\partial t}\right)}{\pi_{0 t} G_{0}+\pi_{1 t} G_{1}} \\
\tilde{\beta_{G}=} & \frac{\pi_{0 t} \beta_{G_{0}} G_{0}+\pi_{1 t} \beta_{G_{1}} G_{1}}{\pi_{0 t} G_{0}+\pi_{1 t} G_{1}} \\
& +\frac{b(t, 1)\left(G_{0}\left(1+\beta_{G_{0}}\right)-G_{1}\left(1+\beta_{G_{1}}\right)\right)}{\pi_{0 t} G_{0}+\pi_{1 t} G_{1}} \\
\tilde{\gamma_{G}=} & \frac{\pi_{0 t} \gamma_{G_{0}} G_{0}+\pi_{1 t} \gamma_{G_{1}} G_{1}}{\pi_{0 t} G_{0}+\pi_{1 t} G_{1}} \\
& +\frac{b(t,-1)\left(G_{0}\left(1+\gamma_{G_{0}}\right)-G_{1}\left(1+\gamma_{G_{1}}\right)\right)}{\pi_{0 t} G_{0}+\pi_{1 t} G_{1}}
\end{aligned}
$$


Proof.

$$
\begin{aligned}
d G= & \pi_{0} d G_{0}+\pi_{1} d G_{1}+G_{0} d \pi_{0}+G_{1} d \pi_{1}+\text { covariance term } \\
= & \pi_{0} d G_{0}+\pi_{1} d G_{1}+\left(G_{0}-G_{1}\right) d \pi_{0}+d\left[G_{0}-G_{1}, \pi_{0}\right] \\
= & -\left(\pi_{0} \frac{\partial G_{0}}{\partial t}+\pi_{1} \frac{\partial G_{1}}{\partial t}\right) d t \\
& +\left(\pi_{0} \beta_{G_{0}} G_{0}+\pi_{1} \beta_{G_{1}} G_{1}\right) d N_{0 t} \\
& +\left(\pi_{0} \gamma_{G_{0}} G_{0}+\pi_{1} \gamma_{G_{1}} G_{1}\right) d N_{1 t} \\
& +\left(G_{0}-G_{1}\right)\left(a(t) d t+b(t, 1) d N_{0 t}+b(t,-1) d N_{1 t}\right) \\
& +\left(\beta_{G_{0}} G_{0}-\beta_{G_{1}} G_{1}\right) b(t, 1) d N_{0 t} \\
& +\left(\gamma_{G_{0}} G_{0}-\gamma_{G_{1}} G_{1}\right) b(t,-1) d N_{1 t} \\
= & \tilde{\alpha} d t+\tilde{\beta} d N_{0 t}+\tilde{\gamma} d N_{1 t}
\end{aligned}
$$

We can hedge an option in the same way as in the fixed $\lambda$ case with $\alpha, \beta, \gamma$ replaced by $\tilde{\alpha}, \tilde{\beta}, \tilde{\gamma}$. We still need one market traded option and the stock to hedge an option. But to get the hedge ratios, we need $\pi(t), a(t), b(t)$.

\section{ESTIMATION}

We use the generalized method of moments to obtain parameter estimates, which is common in finance, specially when options are involved. These are called the 'risk neutral' parameters since they incorporate the price of risk associated with holding options that mature in the future. Prices of options are expectations of discounted terminal payoffs under the risk neutral parameters. So the estimates are obtained by minimizing some distance between the observed price of options and computed expected terminal payoffs under the model.

\subsection{Estimation of constant intensity rate}

We use a Monte Carlo approach for computing the expected terminal payoffs for option prices. We generate the stock price process as follows:

- Generate $\xi_{t}$, the process of waiting times as a homogeneous Poisson process with intensity rate $\lambda$.

- At each event time $S_{t}$ jumps by $\pm c$ with probability $p_{t, N_{t}}$ and $1-p_{t, N_{t}}$.

Generating a large number of samples, we compute the average of $P_{T}$, the payoff of an option at expiration $T$ for different options and expirations. We then compute the mean square error between these and the observed prices of the corresponding options. The value of $\lambda$ that minimizes this error is the GMM estimator.

\subsection{Estimation for stochastic intensity rate}

The parameters involved in this case are: $\pi(t)$ which is the probability that the unobserved intensity process is in state $\lambda_{0}$ at time $t$, the two intensity parameters $\lambda_{0}, \lambda_{1}$, the transition matrix of the intensity process that is determined by $q_{01}, q_{10}$, and $\pi=\pi(0)$. Let $\Theta=\left(\lambda_{0}, \lambda_{1}, q_{01}, q_{10}, \pi\right)$.

We take an Empirical Bayes approach where the hyperparameters $\Theta$ are estimated from the observed price of market traded options. We then update the posterior of the unobserved intensity process by filtering techniques applied on the stock price process.

Inference on the parameter $\pi(t)$ can be done by inverting options at each point of time. Besides involving huge amount of computations this also has the following theoretical drawbacks:

- The hedge ratios involve $a(t), b(t)$. This implies that we need them to be predictable. But if we have to invert an option to get them, then we need to observe the price at time $\mathrm{t}$ to infer $\pi_{t}$ and from there to get $a_{t}$ and $b_{t}$. So they are no more predictable.

- Options are not as frequently traded as stocks and hence option prices are not as reliable as stock prices. Thus, inferring $\pi(t)$ at each time point $t$ by inverting options will give incorrect prices and lead to arbitrage.

So we base our updates only on the stock prices. We describe the various steps involved in this procedure below.

\subsubsection{Monte Carlo approach for pricing options}

The Monte Carlo algorithm to obtain $P_{T}$, the terminal payoff, under fixed values of $\Theta$ is as follows:

- Fix $\theta_{0}=i$.

- Generate the $\theta$ process.

- Generate $\xi_{t}$, the process of waiting times as nonhomogeneous Poisson process with intensity rate $\lambda_{\theta_{t}}$.

- At each event time $S_{t}$ jumps by $\pm c$ with probability $p_{t, N_{t}}$ and $1-p_{t, N_{t}}$.

- Get the expectation under $\theta_{0}=i$ for $i=0,1$.

- Now take the average of these with respect to $\pi_{0}$.

The only difficult step here is generating the $\xi_{t}$ process. This is done as follows:

- Generate $T_{0}$ from $\operatorname{Exp}\left(\lambda_{\theta_{0}} \mathrm{~N}_{0}^{2}\right)$

- Let $\tau_{0}=\inf \left\{t: \theta_{t} \neq \theta_{0}\right\}$

- If $T_{0}<\tau_{0}$, jump at time $T_{0}$.

- Otherwise, generate $T_{1}$ from $\operatorname{Exp}\left(\lambda_{\theta_{\tau_{0}}} \mathrm{~N}_{\tau_{0}}^{2}\right)$

- $\tau_{1}=\inf \left\{t: \theta_{t} \neq \theta_{\tau_{0}}\right\}$

- If $T_{1}<\tau_{1}$, jump at time $\tau_{0}+T_{1}$

- Continue.

This is justified by memorylessness.

\subsubsection{Estimation of hyper-parameters}

The objective is to find the parameter set that minimizes the root mean square error between the observed option price and the computed expected terminal payoff, for all options in the training sample. We followed a diagonally scaled steepest descent algorithm with central difference approximation to the differential. 
The starting values of $\lambda_{0}, \lambda_{1}$ are taken to be equal to the value of the estimator $\hat{\lambda}$ obtained in the constant intensity model.

The starting values of $q_{01}, q_{10}$ are obtained by a hidden Markov model approach using an iterative method that has two steps. Let the underlying state process be $\eta_{t}$, that is the intensity is $\lambda_{i}$ when $\eta_{t}$ is $i$. In one step, the MLE of the parameters is obtained given the $\eta_{t}$ process. For details on the method for obtaining the MLE in this setting, refer to Elliott et al. (1995). Given the latent process the MLE's are obtained from the following equations:

$$
\hat{q}_{i j}=\frac{\text { No. of times latent process jumps from state } i \text { to } j}{\text { Time spent by latent process in state } i}
$$

$$
\sum_{j \in \text { State space }} \frac{\mathcal{A}_{j, j+1}^{m}}{\lambda_{m}+\frac{r}{j}}+\frac{\mathcal{A}_{j, j-1}^{m}}{\lambda_{m}-\frac{r}{j}}-j^{2} \mathcal{T}_{t}^{j, m}=0
$$

where $\mathcal{A}_{i, j}^{m}=$ Number of times observed process jumps from state $i$ to state $j$ when latent process is in state $m$ and $\mathcal{T}_{t}^{j, m}=$ Time during which latent process is in state $m$ and observed process is in state $j$. In the next step, for each $t$ when there is a jump in the stock price, we assign $\eta_{t}$ to that $i$ which maximizes the probability of an event.

We do a finite search on the parameter $\pi$.

Then we perform the minimization algorithm to get the risk neutral parameters.

\subsubsection{Bayesian filtering for inference on the unobserved state process}

As shown in Elliott et al. (1995), the posterior of $\pi_{j}(t)$ is given by:

$$
\begin{aligned}
\pi_{j}(t)= & \pi_{j}(0)+\int_{0}^{t} \sum_{i} q_{i j} \pi_{i}(u) d u \\
& +\int_{0}^{t} \pi_{j}(u)\left(\bar{\lambda}(u)-\lambda_{j}\right) N_{u}^{2} d u \\
& +\sum_{0<u<t} \pi_{j}(u-)\left(\frac{\lambda_{j} p_{\lambda_{j}}\left(S_{u-} \rightarrow S_{u}\right)}{\sum_{i} \pi_{i}(u) \lambda_{i} p_{\lambda_{i}}\left(S_{u-} \rightarrow S_{u}\right)}-1\right)
\end{aligned}
$$

where $\bar{\lambda}(t)=\sum_{i} \pi_{i}(t) \lambda_{i}$.

Thus, $a_{j}(u)=\sum_{i} q_{i j} \pi_{i}(u)+\int_{0}^{t} \pi_{j}(u)\left(\bar{\lambda}(u)-\lambda_{j}\right) N_{u}^{2}$, and $b_{j}(u)=\pi_{j}(u-)\left(\frac{\lambda_{j} p_{\lambda_{j}}\left(S_{u-} \rightarrow S_{u}\right)}{\sum_{i} \pi_{i}(u) \lambda_{i} p_{\lambda_{i}}\left(S_{u-} \rightarrow S_{u}\right)}-1\right)$.

This is the conditional law of the unobserved rate process given the path upto time $t$ of the observed stock price process as in a hidden Markov model.

\subsection{Generalizations}

According to Snyder (1973), the evolution of the $\pi_{i}(t)$ process is given by (4.5). Even if we do not want to specify the prior for the $\lambda$ process, it is still of that form. So the problems that we mentioned in Section 5.2.3 remain, whatever be the prior for the evolution of $\lambda_{t}$.
Let us see the form of the posterior in the general case. Let $c_{t}\left(v \mid N_{t_{0}, t}\right)$ be the posterior characteristic function for $\lambda_{t}$ given an observed path.

$$
\begin{aligned}
d c_{t}\left(v \mid N_{t_{0}, t}\right)= & E\left(\exp \left(i v \lambda_{t}\right) \Psi_{t}\left(v \mid N_{t_{0}, t}, \lambda_{t}\right) \mid N_{t_{0}, t}\right) d t \\
& -E\left(\exp \left(i v \lambda_{t}\right) g\left(N_{t}\right)\left(\lambda_{t}-\hat{\lambda_{t}}\right) \mid N_{t_{0}, t}\right) d t \\
+ & E\left(\exp \left(i v \lambda_{t}\right)\left(\lambda_{N_{t}, N_{t+d t}}-\lambda_{N_{t}, N_{t+d t}}^{*}\right) \mid N_{t_{0}, t}\right) \\
& \times \lambda_{N_{t}, N_{t+d t}}^{*-1} d \xi_{t}
\end{aligned}
$$

$$
\begin{aligned}
\text { where } \lambda_{N_{t}, \zeta_{t}}= & g\left(N_{t}\right) \lambda_{t} p_{\lambda_{t}, N_{t}} \text { if } \zeta_{t}=N_{t}+1 \\
& g\left(N_{t}\right) \lambda_{t}\left(1-p_{\lambda_{t}, N_{t}}\right) \text { if } \zeta_{t}=N_{t}-1 \\
& 0 \mathrm{o} . \mathrm{w} . \\
\lambda_{N_{t}, \zeta_{t}}^{*}= & E\left(\lambda_{N_{t}, \zeta_{t}}\left(t, N_{t_{0}, t}, \lambda_{t}\right) \mid N_{t_{0}, t}\right) \\
\Psi_{t}\left(v \mid N_{t_{0}, t}, \lambda_{t}\right)= & E\left(\exp \left(i v \Delta \lambda_{t}\right) \mid N_{t_{0}, t}, \lambda_{t}\right)
\end{aligned}
$$

For example, let $d \lambda_{t}=f_{t}\left(\lambda_{t}\right) d t+G_{t}\left(\lambda_{t}\right) d W_{t}$, where $W_{t}$ is Brownian motion. In this case $\Psi_{t}\left(v \mid N_{t_{0}, t}, \lambda_{t}\right)=i v f_{t}\left(\lambda_{t}\right)-$ $v^{2} G_{t}^{2}\left(\lambda_{t}\right) / 2$.

As long as the observed process is Markov jump process, the second and third terms are same as in the two state Markov case and hence yield the same function on taking inverse Fourier transform. The first term, on taking inverse Fourier transform yields L, the Kolmogorov-Fokker-Plank differential operator associated with $\lambda$.

\section{REAL DATA APPLICATIONS}

\subsection{Description}

Data on stock price and option price was obtained from the option-metrics database for three stocks Ford, ABMD and IBM. The stock data is transaction by transaction. The format of the raw stock data is: SYMBOL, DATE, TIME, PRICE, SIZE, G127, CORR, COND, EX. After filtering for after hour and international market trading, the data is on tradings in NASDAQ regular hours. The prices are divided by the tick size to obtain integers. We use part of the data as training sample and the rest as test sample. The option data is daily best bid and ask prices. The data has DATE, CALL/PUT, EXPIRATION, BEST-BID, BEST-ASK, STRIKE.

We estimate parameters from the training sample and use these estimates to predict prices of options in the test sample. The 3 stocks provide some variety. The Ford stock is a little old when the tick size used to be $\$ 1 / 16$ while the others have tick size $\$ 1 / 100$. The ABMD data is more thinly traded than the other two. Table 1 presents the dates, sample size, observed range of option prices and the average bidask spread. The figures quoted are in multiples of the tick size. Table 2 presents $\lambda$, average length of predicted interval and the distance measure $a$ for the 2 methods and various datasets. Here $\lambda$ is the volatility for Black-Scholes model and the intensity for the birth-death model. It is observed 
Table 1. Description of data

\begin{tabular}{llrccc}
\hline \hline Data & \multicolumn{1}{c}{ Date } & $\begin{array}{c}\text { Sample } \\
\text { Size }\end{array}$ & $\begin{array}{c}\text { Range of } \\
\text { Option Prices }\end{array}$ & $\begin{array}{c}\text { Bid-ask } \\
\text { spread }\end{array}$ & $\begin{array}{c}\text { Trades } \\
\text { per day }\end{array}$ \\
\hline IBM Training & June 3, 02 & 92 & $0-3700$ & 5 & 4270 \\
IBM Test & June 4-12, 02 & 620 & $0-8000$ & 5 & 4270 \\
ABMD Training & Feb 3, 03 & 12 & $0-160$ & 25 & 400 \\
ABMD Test & Feb 4-28, 03 & 33 & $0-550$ & 25 & 400 \\
Ford Training & Dec 1, 00 & 38 & $0-180$ & 2 & 1675 \\
Ford Test & Dec 5-31, 00 & 578 & $0-256$ & 2 & 1675 \\
\hline
\end{tabular}

All figures are in multiples of the tick size. Sample size denotes the number of different options that are traded: trading date, put/call, strike, expiration combinations. The last column gives the average number of trades per day for that stock over a year.

Table 2. Results for constant intensity rate

\begin{tabular}{lccrcrr}
\hline \hline Model & Dataset & $\lambda$ & \multicolumn{2}{c}{ Training } & \multicolumn{2}{c}{ Test } \\
& & & Length & Distance & Length & Distance \\
\hline BS & IBM & $8.0 \mathrm{e}-07$ & 99.180 & 10.603 & 52.199 & 19.481 \\
BD & IBM & $1.1 \mathrm{e}-06$ & 124.402 & 10.111 & 81.048 & 20.502 \\
BS & ABMD & $6.5 \mathrm{e}-06$ & 9.637 & 1.031 & 12.768 & 3.873 \\
BD & ABMD & $7.5 \mathrm{e}-06$ & 17.540 & 0.973 & 19.465 & 2.431 \\
BS & F & $9.0 \mathrm{e}-07$ & 6.096 & 0.297 & 5.919 & 0.609 \\
BD & F & $1.3 \mathrm{e}-06$ & 6.791 & 0.225 & 6.578 & 0.586
\end{tabular}

For constant intensity rate, this table summarizes the estimated $\lambda$ from the training sample, average length of the predicted interval for option prices and the distance between the observed and predicted intervals.

that the two models provide very similar results. The BlackScholes model gives better prediction for the IBM data. The gain in using the birth-death model is most in the AMBD data possibly because it is thinly traded. There is some gain in the Ford data possibly because of the large tick-size. In both of these cases once we fix the parameter we are considering one fixed model. We get the intervals as the maximum and minimum of the option prices as the stock price varies over the day. So these are not any prediction intervals, but just arise because of intra-day variation in the predicted option price.

In Fig. 2 we present the error (observed-expected) using midpoints of the intervals in pricing of options against strikes conditional on number of days to expiration. It is seen that the CALL options are priced higher by the model. Also we observe the phenomenon of smile that is common to all option pricing models. The top panels are for the learning sample and bottom panels for Fig. 2 the test sample. From the test sample data it is noticed that options that are in the money have higher variability of pricing errors. This is also a common phenomenon of many option pricing models, the cause for which is attributed to higher trading of in the money options than out of the money options.

In Fig. 3 we present the hedging error of the birth-death and the Black Scholes model, both with constant intensity rate. This gives us an idea of the magnitude of gain achieved
Table 3. Evolution of algorithm

\begin{tabular}{ccc}
\hline \hline$\lambda_{0}$ & $\lambda_{1}$ & RMSE \\
\hline $1.100000 \mathrm{e}-06$ & $1.100000 \mathrm{e}-06$ & 52.7742 \\
$1.065162 \mathrm{e}-06$ & $1.594270 \mathrm{e}-07$ & 39.5022 \\
$1.043488 \mathrm{e}-06$ & $8.320846 \mathrm{e}-08$ & 38.2857 \\
$1.042039 \mathrm{e}-06$ & $8.326884 \mathrm{e}-08$ & 29.6799 \\
\hline
\end{tabular}

This table gives the values of the parameters and the root mean square error at consecutive stages of iteration of the algorithm for IBM dataset.

by using the birth-death model. It is seen that considerable gain is achieved by using the birth-death model.

\subsection{Stochastic intensity rate}

For the ABMD and Ford datasets, the RMSE of prediction obtained from the constant intensity method is less than the bid-ask spread. This means that the constant intensity model attains the lower bound on the possible quadratic calibration error as referred to the intrinsic error in Cont (2002). Since the data is observed with an error, that is only as bid-ask quotes, we cannot hope to achieve a level of error less than the precision of the observed data. However for the IBM data there is scope for improvement.

Table 3 shows the values of $\lambda_{0}, \lambda_{1}$ and the RMS error for the evolution of the algorithm. The initial values of $q_{01}, q_{10}$ and $\pi$ are $8.64 \mathrm{e}-02,1.2126$ and 0.5 and they do not change in the significant digits over the evolution of the algorithm.

The algorithm converges with the value of RMSE equal to 29.6799. It is seen that we do achieve substantial improvement in the RMSE by using the stochastic intensity rate model over the birth death model with constant intensity $(\mathrm{RMSE}=52.7729)$. However, the bias and smile that were observed in the constant volatility model still remain for the stochastic volatility model. Also, this is an ill-posed problem in the sense that the solutions are not unique and depend on the starting values of the parameters. There is still scope for improvement since the best RMSE we can hope to attain is the daily bid-ask spread which is 5 in this case. 

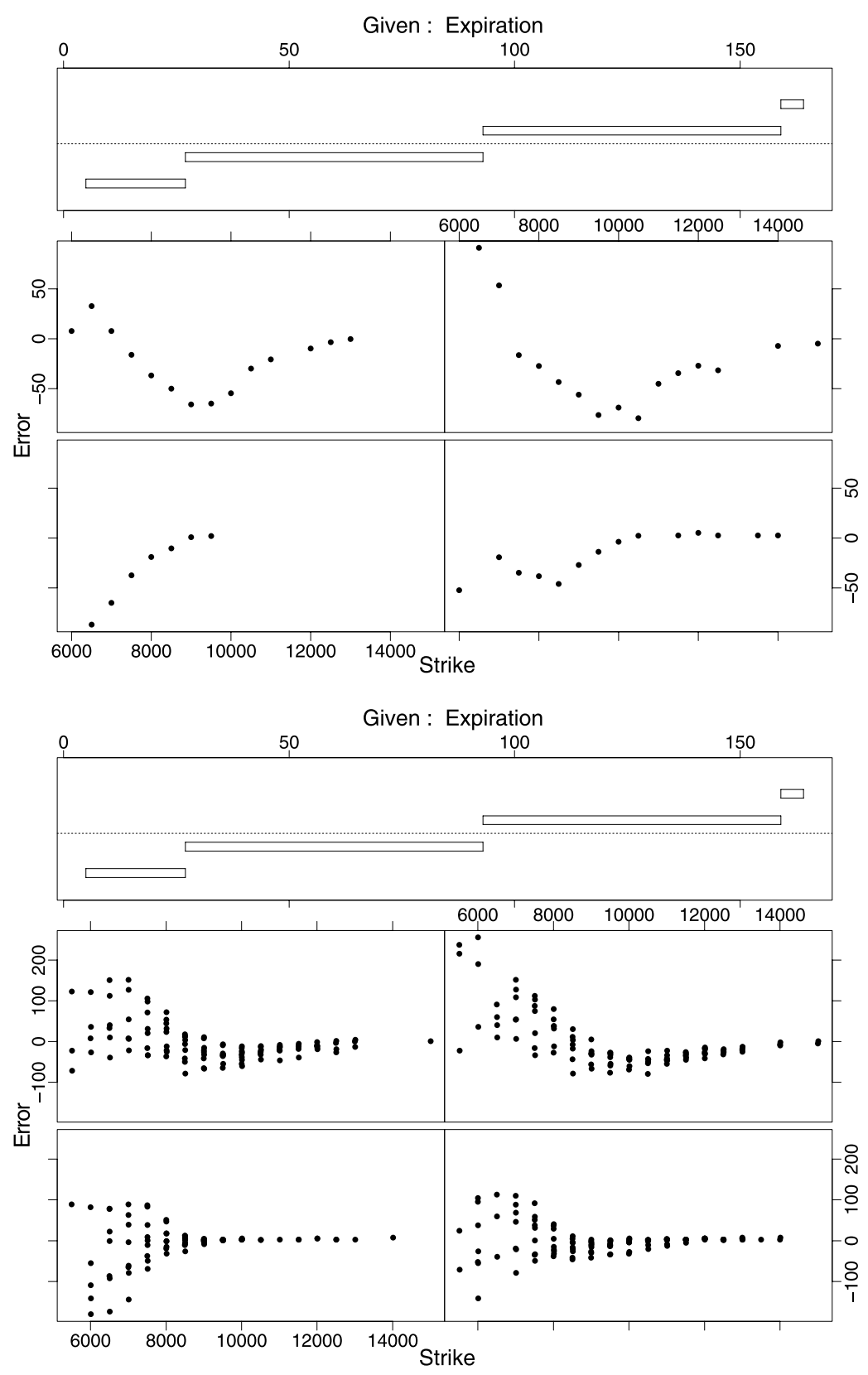

Figure 2. Error in CALL price of IBM data. Top: Training sample, Bottom: Test sample. The four panels are for four Expiration Dates. Inside each panel, we plot the error against the Strike.

\section{CONCLUSIONS}

- Modeling: The birth and death model takes into account the fact that stock prices move in multiples of the tick. This model removes the inconsistency between theory and practice that the continuous path models run into, like quadratic variation being observable.

- Pricing: Both Edgeworth expansion results and study of real data show that the birth and death model with quadratic intensity rate produces option prices that are very similar to the Black-Scholes model. In a way, this study explains why the industry practice of using the Black-Scholes model works in practice despite of being adhoc and contradicting the underlying theory.

- Hedging: The birth and death model is different from general models with jumps in the capacity that one can set up derivative security hedging in this model. The hedging is qualitatively and significantly different from the Black-Scholes model. One needs not only the stock and bond, but another market traded option to hedge. This is analogous to the industry practice of gamma hedging. Considerable monetary gain is attained by us- 


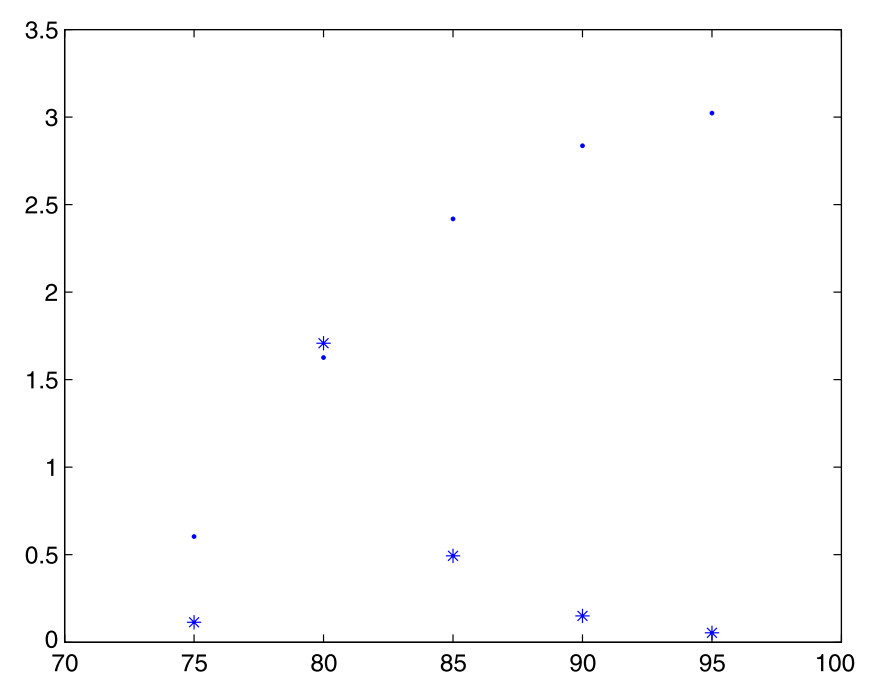

Figure 3. Hedging error for the IBM data-set. For Put-options expiring on July 22, 2002, we obtain the hedging errors (in real dollars) for holding the portfolio from July 1-July 22.

The error obtained by hedging using the Black-Scholes model is shown with dots and that with the Birth-Death model is shown with stars. The $x$-axis is the strike price.

ing the birth and death model for hedging as opposed to the Black-Scholes model. Also, the introduction of stochastic volatility does not necessitate the introduction of extra derivative securities for hedging purposes.

\section{APPENDIX A. PROOF OF THEOREM}

Define $X_{t}^{(n)}=\ln \left(\frac{N_{t}^{(n)}}{n}\right)$ and

$$
\begin{aligned}
X_{t}^{*(n)}= & X_{t}^{(n)}-\int_{0}^{t}\left[p_{u} \log \left(1+\frac{1}{N_{u}}\right)\right] \\
& +\int_{0}^{t}\left[\left(1-p_{u}\right) \log \left(1-\frac{1}{N_{u}}\right)\right] N_{u}^{2} \sigma_{u}^{2} d u-X_{0}^{(n)}
\end{aligned}
$$

where $p_{t, N_{t}}=\frac{1}{2}\left(1+\frac{r_{t}}{N_{t} \sigma_{t}^{2}}\right)$.

Lemma 1. $\forall t>0$, $\left[p_{t, N_{t}} \log \left(1+\frac{1}{N_{t}}\right)+\left(1-p_{t, N_{t}}\right) \log \left(1-\frac{1}{N_{t}}\right)\right] N_{t}^{2} \sigma_{t}^{2} \stackrel{P}{\longrightarrow} r_{t}-\frac{\sigma_{t}^{2}}{2}$. Proof. $\left[p_{t, N_{t}} \log \left(1+\frac{1}{N_{t}}\right)+\left(1-p_{t, N_{t}}\right) \log \left(1-\frac{1}{N_{t}}\right)\right] N_{t}^{2} \sigma_{t}^{2}=$ $\left(2 p_{t, N_{t}}-1\right) N_{t}^{2} \sigma_{t}^{2} \sum_{k=1}^{\infty} \frac{1}{(2 k-1) N_{t}^{2 k-1}}-N_{t}^{2} \sigma_{t}^{2} \sum_{k=1}^{\infty} \frac{1}{2 k N_{t}^{2 k}}=$ $r_{t}-\frac{\sigma_{t}^{2}}{2}+r_{t} \sum_{k=1}^{\infty} \frac{1}{(2 k+1) N_{t}^{2 k}}-\sigma_{t}^{2} \sum_{k=1}^{\infty} \frac{1}{(2 k+2) N_{t}^{2 k}} \stackrel{P}{\longrightarrow}$ $r_{t}-\frac{\sigma_{t}^{2}}{2}$

Lemma 2. $X_{t}^{*(n)}$ is a local martingale.

Proof. Let $d N_{1 t}=I_{Y_{t}=+1} d N_{t}$ and $d N_{2 t}=-I_{Y_{t}=-1} d N_{t}$ with $N_{i 0}=0$. $\left(N_{1 t}, N_{2 t}\right)$ is 2-dimensional point processes with $d N_{t}=d N_{1 t}-d N_{2 t}$ and intensity $\left(\lambda_{1 t}:=\sigma_{t}^{2} p_{t, N_{t}} N_{t}^{2}, \lambda_{2 t}:=\right.$ $\left.\sigma_{t}^{2}\left(1-p_{t, N_{t}}\right) N_{t}^{2}\right)$

478 R. Sen
$Q_{i t}=N_{i t}-\int_{0}^{t} \lambda_{i s} N_{s}^{2} d s$ is the compensated point process associated with $N_{i t}$ and hence by Theorem T8 of Bremaud $(1981) X_{t}^{*(n)}=\int_{0}^{t} \log \left(1+\frac{1}{N_{t}}\right) d Q_{1 t}+\int_{0}^{t} \log \left(1-\frac{1}{N_{t}}\right) d Q_{1 t}$ is a local martingale if $\int_{0}^{1}\left|\ln \left(1+\frac{1}{N_{t}}\right)\right| N_{t}^{2} \lambda_{t} d t$ and $\int_{0}^{1} \mid$ $\ln \left(1-\frac{1}{N_{t}}\right) \mid N_{t}^{2} \lambda_{t} d t$ are finite a.s.

Lemma 3. $\left[X_{t}^{*(n)}, X_{t}^{*(n)}\right]_{t} \stackrel{P}{\longrightarrow} \int_{0}^{t} \sigma_{u}^{2} d u \quad \forall t \in D$ dense.

Proof. Using the same technique as in step (2), we can show that: $\int_{0}^{t} \log \left(1+\frac{1}{N_{t}}\right)^{2} d Q_{1 t}+\int_{0}^{t} \log \left(1-\frac{1}{N_{t}}\right)^{2} d Q_{1 t}$ is a local martingale if $\int_{0}^{1}\left|\ln \left(1+\frac{1}{N_{t}}\right)^{2}\right| N_{t}^{2} \sigma_{t}^{2} d t$ and $\int_{0}^{1}\left|\ln \left(1-\frac{1}{N_{t}}\right)^{2}\right|$ $N_{t}^{2} \sigma_{t}^{2} d t$ are finite a.s.

Hence,

$$
\begin{aligned}
\frac{d}{d t} & <X^{*(n)}, X^{*(n)}>_{t} \\
& =\left[p_{t, N_{t}}\left\{\log \left(1+\frac{1}{N_{t}}\right)\right\}^{2}+\left(1-p_{t, N_{t}}\right)\left\{\log \left(1-\frac{1}{N_{t}}\right)\right\}^{2}\right] N_{t}^{2} \sigma_{t}^{2}
\end{aligned}
$$

An expansion similar to the one in Lemma 1 shows that this quantity converges in probability to $\sigma_{t}^{2}$ for all $t>0$.

$$
\text { Let } M_{t}^{(n)}=\left[X_{t}^{*(n)}, X_{t}^{*(n)}\right]-<X^{*(n)}, X^{*(n)}>_{t}
$$

if Assumption 2.2 holds, $\left[M^{(n)}, M^{(n)}\right]_{t}=\sum_{\tau_{i} \leq t}\left|\Delta X_{\tau_{i}}^{(n)}\right|^{4} \stackrel{P}{\longrightarrow} 0$ and the result follows.

$$
\left|\Delta X_{t}^{*(n)}\right|=\log \left(1+\frac{Y_{t}}{N_{t}}\right) \leq \log (2) \quad \text { identically. }
$$

Lemma 2, Lemma 3, (A.1) and Theorem VIII.3.11 of Jacod and Shiryaev (2003) imply $X^{*(n)}$ converges in law to a continuous Gaussian Martingale with characteristics $\left(0, \int_{0}^{t} \sigma_{u}^{2} d u, 0\right)$. This and Lemma 1 imply $X^{(n)}$ converges in law to $X$, a continuous Gaussian Martingale with characteristics $\left(\int_{0}^{t}\left(r_{u}-\frac{1}{2} \sigma_{u}^{2}\right) d u, \int_{0}^{t} \sigma_{u}^{2} d u, 0\right)$.

\section{APPENDIX B. EDGEWORTH EXPANSION}

We shall use the Theorem 1, conditions (I1-3) and Proposition 3 from Mykland (1995). Let us consider $\ell_{t}^{n}=\sqrt{n} X_{t}^{*(n)}$ where $X_{t}^{*(n)}$ is defined in Appendix A. Let $\kappa=\lambda T, T_{n}=T$, $c_{n}=n, r_{n}=1 / n$. We shall suppress the $i$ in the notation, because we are in the univariate case. Then

$$
\begin{aligned}
{\left[\ell^{n}, \ell^{n}, \ell^{n}, \ell^{n}\right]_{T_{n}} } & =n^{2} \int_{0}^{T}\left[\log \left(1+\frac{Y_{u}}{N_{u-}}\right)\right]^{4} d \xi_{u} \\
\mathrm{E}\left[\ell^{\mathrm{n}}, \ell^{\mathrm{n}}, \ell^{\mathrm{n}}, \ell^{\mathrm{n}}\right]_{\mathrm{T}_{\mathrm{n}}} & =n^{2} \int_{0}^{T} \mathrm{E}\left[\left(\frac{1}{\mathrm{~N}_{\mathrm{u}-}}\right)^{4} \mathrm{~N}_{\mathrm{u}}^{2} \lambda\right] \mathrm{du}+\mathrm{o}(1) \\
& =\int_{0}^{T} \mathrm{E}\left(\frac{1}{\mathrm{~S}_{\mathrm{u}-}^{2}}\right) \lambda \mathrm{du}+\mathrm{o}(1) \\
& =O\left(c_{n}^{2} r_{n}^{2}\right)
\end{aligned}
$$


So condition (I3) is satisfied.

$$
\begin{aligned}
& \frac{<\ell^{n}, \ell^{n}, \ell^{n}>_{u T_{n}}}{c_{n}^{3 / 2} r_{n}} \\
& =\frac{n^{3 / 2}}{\sqrt{n}} \int_{0}^{T} \mathrm{E}\left[\left\{\ln \left(1+\frac{\mathrm{Y}_{\mathrm{u}}}{\mathrm{N}_{\mathrm{u}-}}\right)\right\}^{3} \mid \mathcal{F}_{\mathrm{u}-}\right] \mathrm{N}_{\mathrm{u}-}^{2} \lambda \mathrm{du} \\
& =\frac{1}{n}\left(r-\frac{3 \lambda}{2}\right) \int_{0}^{T} \frac{1}{S_{u-}^{2}} d u+o(1 / n)
\end{aligned}
$$

This implies $\eta$ of Proposition 3 in Mykland (1995) is 0. Hence we can replace $\left(\ell^{n}, \ell^{n}\right)_{T_{n}}$ by $<\ell^{n}, \ell^{n}>_{T_{n}}$ in the definition of $\varrho$.

$$
\begin{aligned}
& <\ell^{n}, \ell^{n}>_{T} / n \\
& =\int_{0}^{T} \mathrm{E}\left[\left\{\ln \left(1+\frac{\mathrm{Y}_{\mathrm{u}}}{\mathrm{N}_{\mathrm{u}}}\right)\right\}^{2} \mid \mathcal{F}_{\mathrm{u}-}\right] \mathrm{N}_{\mathrm{u}-}^{2} \lambda \mathrm{du} \\
& =\int_{0}^{T}\left[p_{u, N_{u-}}\left\{\ln \left(1+\frac{1}{N_{u-}}\right)\right\}^{2}\right] N_{u-}^{2} \lambda d u \\
& \quad+\left[\left(1-p_{u, N_{u-}}\right)\left\{\ln \left(1+\frac{1}{N_{u-}}\right)\right\}^{2}\right] N_{u-}^{2} \lambda d u \\
& =\int_{0}^{T}\left[\frac{1}{N_{u-}^{2}}+\frac{1-2 p_{u, N_{u-}}}{N_{u-}^{3}} \frac{2}{3 N_{u-}^{4}}+\frac{1}{4 N_{u-}^{4}}\right] N_{u-}^{2} \lambda d u \\
& +\operatorname{smaller} \text { order terms } \\
& =\lambda T+\left(\frac{2}{3}+\frac{1}{4}-\frac{r}{\lambda}\right) \lambda \int_{0}^{T} \frac{1}{N_{u-}^{2}} d u+\mathrm{smaller}^{2} \text { order terms } \\
& \varrho=\mathrm{E}_{\mathrm{as}}\left(\mathrm{n}\left(\frac{<\ell^{\mathrm{n}}, \ell^{\mathrm{n}}>_{\mathrm{T}}}{\mathrm{n}}-\lambda \mathrm{T}\right) \mid \mathrm{X}_{\mathrm{T}}^{*(\mathrm{n})}\right) \\
& \quad=\left(\frac{11}{12} \lambda-r\right) \mathrm{E}_{\mathrm{as}}\left(\frac{1}{\mathrm{n}} \int_{0}^{\mathrm{T}} \frac{1}{\mathrm{~S}_{\mathrm{u}-}^{(\mathrm{n}) 2}} \mathrm{du} \mid \mathrm{X}_{\mathrm{T}}^{*(\mathrm{n})}\right) \\
& \quad=0
\end{aligned}
$$

Hence, if there exist constants $\underline{k}, \bar{k}$ satisfying assumptions (I1) and (I2), then for any $g \in \mathcal{C}$,

$$
\operatorname{Eg}\left(\mathrm{X}_{\mathrm{T}}^{*(\mathrm{n})}\right)=\operatorname{Eg}(\mathrm{N}(0, \lambda \mathrm{T}))+\mathrm{o}(1 / \mathrm{n}) .
$$

\section{Received 16 January 2009}

\section{REFERENCES}

Andersen, T. G., Bollerslev, T., Diebold, F. X., and Labys, P. (2001). "The distribution of realized exchange rate volatility." Journal of American Statistical Association 96(453) 42-55. MR1952727

Bakshi, G., CaO, C., and Chen, Z. (1997). "Empirical Performance of Alternative Option Pricing Models." The Journal of Finance LII(3) 2003-2049.

BATES, D. (1996). "Jumps and stochastic volatility: Exchange rate processes implicit in deutsche mark options." The Review of Financial Studies 9(1) 69-107.
Black, F. and Scholes, M. (1973). "The Pricing of Options and Corporate Liabilities." The Journal of Political Economy 81(3) 637654.

Cont, R. (2002). "Dynamics of implied volatility surfaces." Quantitative Finance 2(1) 45-60. MR1919585

Dengler, H. and JARrow, R. (1997). "Option pricing using a binomial model with random time steps (a formal model of gamma hedging)." Review of Derivatives Research 1 107-138.

Duffie, D., Pan, J., and Singleton, K. (2000). "Transform analysis and asset pricing for affine jump-diffusions." Econometrica 68(6) 1343-1376. MR1793362

Elliott, R. J., Aggoun, L., and Moore, J. B. (1995). "Hidden Markov models." Applications of Mathematics (New York) 29. Springer-Verlag, New York. MR1323178

Engle, R. and Mustafa, C. (1992). "Implied arch models from option prices." Journal of Econometrics 52 289-311.

JACOD, J. and Shiryaev, A. N. (2003). "Limit theorems for stochastic processes." Grundlehren der Mathematischen Wissenschaften [Fundamental Principles of Mathematical Sciences] 288. SpringerVerlag, Berlin. MR1943877

Hayashi, T. and Mykland, P. A. (2005). "Evaluating hedging errors: An asymptotic approach." Mathematical Finance 15 309-343. MR2132193

Hubalek, F., Kallsen, J., and KrawczyK, L. (2006). "Varianceoptimal hedging for processes with stationary independent increments." Annals of Applied Probability 16(2) 853-885. MR2244435

Hull, J. C. and White, A. (1987). "The pricing of options on assets with stochastic volatilities." Journal of Finance 42 281-300.

Korn, R., Kreer, M., and Lenssen, M. (1998). "Pricing of European options when the underlying stock price follows a linear birth-death process." Communications in Statistics. Stochastic Models 14(3) 647-662. MR1621350

LARge, J. (2005). "Estimating Quadratic Variation When Quoted Prices Change by a Constant Increment." Nuffield College Economics Group Working Paper.

Leippold, M. (2002). "Asset Pricing under the Quadratic Class." The journal of financial and quantitative analysis 37(2) 271-295.

MYKLAND, P. A. (1995). "Martingale expansions and second order inference." The Annals of Statistics 23(3) 707-731. MR1345195

MYKLAND, P. A. (1996). "Option pricing and statistical uncertainty." Technical Report 430, Department of Statistics, University of Chicago.

Mrkland, P. A. (2000). "Conservative delta hedging." Annals of Applied Probability 10 664-683. MR1768218

NAIK, V. (1993). "Option valuation and hedging strategies with jumps in the volatility of asset returns." The Journal of Finance 68(5) 1969-1984.

Piazzesi, M. (2001). "An Econometric Model of the Yield Curve with Macroeconomic Jump Effects." NBER Working Paper 8246.

Russell, J. R. and Engle, R. F. (2005). "A discrete-state continuoustime model of financial transactions prices and times: The ACMACD model." Journal of Business \& Economic Statistics 23 166180. MR2157268

SCHWEIzer, M. (1992). "Mean-Variance Hedging for General Claims." The Annals of Applied Probability 2(1) 171-179. MR1143398

SEN, R. (2006). "Intervals for option prices." International Journal of Statistics and Management Systems 1(1) 59-82. MR2340913

SNYDER, D. L. (1973). "Information processing for observed jump processes." Information and Control 22 69-78. MR0326960

\section{Rituparna Sen}

Department of Statistics

University of California at Davis

4218 Mathematical Sciences Building

One Shields Ave., Davis, CA 95616, USA

Phone: 530-752-7623; Fax: 530-752-7099

E-mail address: rsen@ucdavis.edu 